\title{
ON BEING AT HIGHER RISK A QUALITATIVE STUDY OF PRENATAL SCREENING FOR CHROMOSOMAL ANOMALIES
}

Heyman B., Lewando Hundt G., Sandall J., Spencer K., Williams C., Grellier R., and Pitson L. (2006) On being at higher risk: A qualitative study of prenatal screening for chromosomal anomalies. Social Science \& Medicine, 62, 2360-72. 


\section{ABSTRACT}

This paper explores the meaning of higher risk status to women undergoing prenatal maternal screening for chromosomal anomalies. Quotations from lightly structured interviews and transcripts of pre-screening consultations are used to illustrate pregnant women's diverse responses to the offer of screening, and to entering, living with and exiting from higher risk status. Some women reject screening in order to avoid the psychosocial and medical risks associated with higher risk status, or because they rule out pregnancy termination. They may question the risk selection built implicitly into the provision of preventative systems for some health problems but not others. Women who screen at higher risk may challenge this designation by questioning the system-specific probability used to separate them from the lower risk population. However, some experience distress even when they appreciate the precautionary basis on which their higher risk designation is based. They may find disengagement from higher risk status difficult after a diagnostic test has ruled out chromosomal anomalies. The findings highlight the complexity of communicating risk information to pregnant women and other screened populations, the need to support those living with higher risk status and the benefits of keeping the time lived with this status as short as possible.

200 words 


\section{KEY WORDS}

Chromosomal anomalies

Maternity care

Risk communication

Risk management

Prenatal Screening

Qualitative research 


\section{INTRODUCTION}

This paper will explore women's understandings of higher risk health status, drawing upon a study of prenatal screening for chromosomal anomalies. Higher risk status is constituted by screening systems operating at the limits of technological innovation where non-invasive and individually accurate tests are not yet available. At this frontier, non-invasive screening systems which divide populations into categories with lower and higher probabilities of experiencing a health problem may be developed. Those who screen above a defined risk threshold can then be offered accurate but invasive diagnostic tests. Higher risk status, although not the conditions tested for, is entirely constituted by the screening system. Screened individuals may realise that the results will change 'their' probability of encountering the selected problem, but cannot know the direction of this change.

Ethical issues concerning abortion and disability (Williams, Alderson and Farsides, 2002) would remain even if an affordable, non-invasive and accurate diagnostic test for chromosomal anomalies were available. Screening offers a second-best, probabilistic view of the unknown chromosomal status of the fetus, derived from empirical associations, usually neither causal or understood, between diverse markers and the screened condition. Since each marker differentiates populations with a greater prevalence of the condition, combining markers will improve prediction providing that the markers are not themselves closely associated. Probabilistic induction from populations to individuals requires heuristic acceptance of the ecological fallacy that aggregate properties of a category appertain to its members (Robinson, 1950; Greenland and Robbins, 1994; Heyman, Henriksen and Maughan, 1998).

Heuristics offer simple rules concerning search, search termination and decisionmaking in complex environments (Gigerenzer and Selten, 2001, p. 8). Heuristics mostly work well enough, but will not always provide a sound guide to action, the price paid for simplification. The probability heuristic will assign some individuals to the higher risk group who do not have the health problem in question and vice versa. The proportions of cases located in these two undesirable categories will depend upon the accuracy of the screening indicators used and the cut-off probability employed for differentiating lower from higher risk cases. With infrequent events, even a low percentage of higher risk cases leads to a large proportion of cases being located in this category despite not having the index condition (Gigerenzer 2003). Table 1 illustrates this problem with respect to first trimester screening for Down's syndrome and other less common chromosomal anomalies (Bindra et al., 2002). 
Table 1: First Trimester Screening for Chromosomal Anomalies (Bindra et al, 2002)

\begin{tabular}{|c|r|r|r|r|}
\hline & fetus has DS & $\begin{array}{r}\text { fetus has other } \\
\text { chromosomal } \\
\text { anomaly }\end{array}$ & $\begin{array}{r}\text { fetus does not } \\
\text { have chromosomal } \\
\text { anomaly }\end{array}$ & TOTAL \\
\hline $\begin{array}{c}\text { Higher risk } \\
\text { screening }(>1: 300)\end{array}$ & 75 & 54 & 967 & 1096 \\
\hline $\begin{array}{c}\text { Lower risk } \\
\text { screening }(<1: 300)\end{array}$ & 7 & 7 & 13273 & 13287 \\
\hline TOTAL & $\mathbf{8 2}$ & 61 & $\mathbf{1 4 2 4 0}$ & $\mathbf{1 4 3 8 3}$ \\
\hline
\end{tabular}

Table 1 shows that the positive predictive value, the proportion of true cases among those classified as being at higher risk as about $12 \%(75+54 / 1096)$ for this system. Although it is more accurate than second trimester screening (e.g. Canini et al., 2002), about $90 \%$ of women who screen at higher risk will not be carrying a fetus with chromosomal anomalies. Because maternal age is included in the risk calculation, older women face a much lower positive predictive value than do younger women, but are less likely to screen at lower risk if carrying a fetus with chromosomal anomalies (Spencer, 2001).

Suggestions for improving the predictive power of this screening system, for example by assessing structural heart defects (Fredouille et al., 2002), are frequently put forward. However, increasing the number of markers may reduce the overall reliability of risk estimation as each marker may be affected by measurement error (Seth and Ellis, 1994). Combining first and second trimester screening, as recommended by Wald, Watt and Hackshaw (1999), would generate more accurate predictions but leave women waiting several weeks longer for their results. Prediction can also be improved by taking into account covariates of the screening markers, for example a previous higher risk classification in the absence of chromosomal anomalies, and a history of maternal smoking (Aitken et al., 2003). Because of the atheoretical, weakly correlational nature of this form of knowledge, new candidate markers and covariates will be continually identified, subjecting established screening systems to constant pressure to change.

The social status of being at higher risk is constituted entirely by screening provision. Its costs are psychosocial and medical. A recent systematic review of the extensive available literature (Green et al., 2004) concluded that women, particularly younger women, experience an increase in anxiety after screening at higher risk which is not necessarily assuaged by a negative diagnosis. One possible explanation for the latter finding is that screening at higher risk for chromosomal anomalies makes the risk of other adverse outcomes more salient. Conflicting research conclusions have been drawn as to whether serious psychological reactions are common (Leithner et al., 2004) or uncommon (Goel et al., 1998). The longer term impact of living temporarily with higher risk status is not known. Detection of higher risk through a scan rather than chemical markers may cause more anxiety (Weinans et al., 2004), perhaps because it is associated with viewing the fetus. Green et al. (2004) hedge their conclusions with 
methodological cautions, for example that more anxious women may exclude themselves from studies by declining to complete questionnaires. Moreover, they reference average responses. Qualitative data, including our own, discussed below, documents the crucial mediating role of women's interpretations of being at higher risk.

Available accurate but invasive diagnostic tests, chorionic villus sampling (CVS) and amniocentesis, are associated with medical risks, including that of spontaneous abortion (Tabor et al., 1986). The identified increase in risk is small, from $0.7 \%$ to $1.7 \%$ in the above large, randomised controlled trial of second trimester amniocentesis versus ultrasound screening, but is faced by all women who undergo diagnostic tests. Most will not be carrying a fetus with chromosomal anomalies. The chromosomal screening and testing system operates as an upwards risk escalator, since an initial risk assessment can lead to further interventions which in turn generate new psychosocial and medical risks (Heyman, 2005).

The present paper will consider women's perspectives on becoming candidates for higher risk status, living with this status, and exiting from it when chromosomal anomalies are ruled out.

\section{METHODOLOGY}

Data were collected at two hospitals in suburban London selected to be comparable in terms of the socioeconomic and ethnic profiles of their surrounding populations. Both offer universal maternal prenatal screening for chromosomal anomalies. One hospital, the 'standard site', provides second trimester screening based on maternal age and serum testing. Those with an estimated probability of carrying a fetus with chromosomal anomalies greater than 1:250 are offered a diagnostic test, usually amniocentesis. Women have to wait one week for screening, and a further 10-21 days for diagnostic test results unless they pay for private testing. The survey indicated that that about twothirds of respondents underwent screening, and that about two-thirds of these women opted to pay for private nuchal translucency thickness screening via an ultrasound scan (Lewando Hundt et al., 2005).

The 'innovative site' provides first trimester screening based on nuchal thickness, maternal age and serum testing. Women receive a pre-test consultation, screening and a post-test consultation about their combined risk estimate at a single one hour visit. Women whose probability of carrying a fetus with chromosomal anomalies is estimated to be greater than 1:300 are offered a diagnostic test, amniocentesis or CVS, with results delivered within one week. The innovative site generates more accurate screening results than the standard site. This gain is taken as a reduced proportion of chromosomal anomalies within the lower risk category for the same $(5 \%)$ rate of women classified as at higher 
risk. The survey data indicated higher screening uptake at the innovative than at the standard site (Lewando Hundt et al., 2005).

The research protocol was approved by the two local research ethics committees. Participants received an information sheet, and gave informed consent to be included in the study. The study design included surveys, interviews and transcription of consultations. The present paper draws primarily on lightly structured multiple interviews with 27 women, undertaken at different stages of their pregnancy, as summarised in Table 2, below.

\section{TABLE 2: Summary of Interview Sampling ( $\mathrm{N}$ of respondents=27)}

\begin{tabular}{|c|c|c|c|}
\hline SITE & Innovative & Standard & TOTAL \\
\hline Pre-screening $^{1}$ & 3 & 7 & 10 \\
\hline Post-screening/diagnostic testing ${ }^{2}$ & 14 & 7 & 21 \\
\hline Postnatal & 11 & 9 & 20 \\
\hline TOTAL & 28 & 23 & 51 \\
\hline
\end{tabular}

\footnotetext{
${ }^{1}$ Before any screening would have occurred at that site.

${ }^{2}$ After any screening/diagnostic testing would have been completed and results communicated, except in two cases, where results were not yet fully known.
}

The ethnicity, pregnancy and age profiles of the interview sample match those of the cachement areas for the hospital research sites (Lewando Hundt et al., 2005). Three respondents were from non-White ethnic groups, and 17 were undergoing their first pregnancy. Five interviewees were aged over 35, putting them roughly into the current higher risk category on the basis of their age related probability $(1: 310)$ of chromosomal anomalies alone. Two of these older women screened at lower risk, two screened at higher risk and accepted diagnostic testing, and one opted for diagnostic testing without screening on account of her age (40). Four younger women, aged 34 or younger, screened at higher risk. Two of these women declined diagnostic testing.

After the pre-screening interviews had been completed, additional respondents were purposively sampled for post-screening/diagnostic testing interviews to cover different screening pathways. The full sample included: screening declined (5); screened at lower risk (14); screened at higher risk but declined diagnostic testing (2); received a diagnostic test (5); and miscarried before screening (1). These pathways were represented at each site. No chromosomal anomalies were diagnosed within the interview sample. Because of the low numbers in most of these groups, the findings should be regarded as illustrative rather than representative. Two transcripts of consultations about chromosomal risks will also be drawn upon, but analysis focuses mainly on women's retrospective views of the screening/diagnosis process. Overall, the sampling design allowed screening pathways to be explored prospectively and retrospectively at different pregnancy stages.

Hospital staff sent out introductory letters and information leaflets with the first appointment booking letter. Women were then approached personally at the 
clinic. Approximately half declined to participate at each site. Respondents were offered a choice of location, with most interviews taking place at their home. The interviews, which mostly lasted 60-90 minutes, covered a range of pregnancy related issues, but the present paper focuses on discussions of chromosomal screening and diagnostic testing. Consultations with a health care practitioner, usually a midwife, were tape-recorded, or transcribed using shorthand if consent to tape-record was not given. Only about $20 \%$ of women approached agreed to their consultation being taped. The interviews and consultations are not necessarily representative, but allowed a range of views to be explored.

Inevitably, the reported study outcomes reflect the researchers' selection of data.

The qualitative interview data and consultation recordings were analysed thematically in relation to women's understandings of risk management for chromosomal anomalies. The categorisation was influenced by a previous study of risk management in prenatal chromosomal screening (Heyman and Henriksen, 2001). The approach adopted fits well with framework analysis (Ritchie and Lewis, 2003), i.e. systematic consideration of themes derived from the data. Data interpretations were discussed within the research team. However, the validity of the qualitative analysis offered needs to be judged by the reader.

\section{DATA ANALYSIS}

Data analysis explored the beginning, middle and end of women's encounters with higher risk status, in relation to the following four questions. How do risk considerations affect pregnant women's decisions to accept or reject screening? How do screened women manage the period of higher risk candidacy? What does being at higher risk mean to those who temporarily acquire this status? How do women understand their exit from higher risk status?

Information about each data extract, provided at the end of each quotation, includes a numerical case identifier indicating the site (1 innovative and 2 standard) with a unique identifier following the decimal point (e.g. 2.4), the participant's age, a brief indicator of the screening decision/outcome and the data source. With respect to screening outcomes, women were informed that they were at lower or higher risk of chromosomal anomalies depending on whether the overall probability of this outcome was below or above the threshold chosen by the screening site managers for recommending diagnostic testing (1:250 and 1:300 at the standard and innovative sites as outlined above).

\section{Screening Decision-Making}

\section{Acceptance of Higher Risk Candidacy}

Women who were screened and informed of their results faced a five percent probability that they would screen at higher risk. Hence, all screened women became candidates for higher risk status, which most would not acquire. Themes 
associated with acceptance of screening included routinisation of the procedure, use of a heuristic of trust to simplify complex probabilistic reasoning, engagement with the maternity care system through screening, and selective attention to screened for conditions. Exceptionally, women acquired higher risk status against their wishes.

Some women accepted screening without question, even though this decision placed them at risk of acquiring higher risk status. The survey showed that a much higher proportion of women attending the innovative site than of those attending the standard site agreed with the statement that screening was part of routine care, i.e. that its acceptance was unproblematic, and that it was assumed that they would be having it (Lewando Hundt et al., 2005). The following discussion of a taped consultation illustrates how routine acceptance of screening for chromosomal anomalies could be accomplished.

Screening is introduced in the context of, as the midwife puts it, 'what we call a low risk pregnancy'. This context may lead women to conclude that their chromosomal screening status will also be low risk. Having outlined the screening procedure, the midwife describes its probabilistic logic.

Midwife: And then the computer churns out a piece of paper that says this woman's risk is one in a hundred, one in 1000, one in 10,000. It doesn't diagnose. That's the main thing to stress. It doesn't come out and say this woman has got a baby with an abnormality. It just puts into category of risk. Pregnant woman: Right.

Midwife: Anybody with a risk of between 1 in 300 is considered by us to be what we call slightly higher risk than the national average. So we then talk to you again and give you the chance to have further tests.

Pregnant woman: Right.

Midwife: But ... if we came along and said, 'You've come back, and the risk is 1 in 280', that still means it's fairly low ... So the main thing is to stress, don't get into a panic if you do decide to be screened and you get a recall, because, for every 100 women we recall, only five will have a baby with a problem. The other 95 will have a time of anxiety, but they wont be a problem, so -

Pregnant woman: Right.

Midwife: Do you think you want to have the screening done?

Yeah. (Booking consultation 1.4, age not known, accepted screening, innovative site)

This quotation illustrates the complexity of the probabilistic reasoning which health professionals attempt to communicate to service users. The cut-off of 1:300 used in this centre to differentiate lower from higher risk is justified in relation to the 'national average'. This interpretation naturalises the selected cutoff, obscuring its origin in a system-specific tradeoff between test sensitivity and specificity. The emphasis placed on computerised calculations, as against the 
assumptions behind them, gives the decision-making process an aura of objective description. The reference to a 5:100 'recall' rate appears to confuse the overall risk of receiving a higher risk test result $(5 \%)$ with the risk of having a fetus with chromosomal anomalies given a higher risk screening result. The best estimate of the latter is a woman's screening result.

Qualitative analysis documents women's reasons for accepting screening. The unquestioning acceptance of screening is illustrated below.

Interviewer: Why did you decide to have the screening test ...

Pregnant woman: There's no reason to say no. Anyway the blood was fine, and you might as well, yeah. (1.4, aged 28, screened lower risk, innovative site, postnatal interview)

Although this respondent justified her acceptance of screening in terms of her lower risk status, she could not have anticipated this outcome when she made her decision. The aside of 'you might as well' suggests that health services should be accepted if offered. In turn, this view is predicated on the trust which some women placed in the beneficence of the NHS.

Who would really get into so much detail to find out [about screening] even though my family, like my dad is a consultant anaesthetist, and my mother a dentist? But you just ... basically trust [name of consultant], and whatever is to be done, then just do it. (1.4, aged 28 , screened lower risk, innovative site, post-screening interview)

This woman employed trust in medicine as a heuristic substitute for engaging with the complex 'detail' of probabilistic inference. However, she may not have appreciated the cost of accepting screening, namely acceptance of the risk of acquiring higher risk status in the absence of chromosomal anomalies.

Another feature potentially linking the mere offer of screening to its acceptance is its association to engagement with the maternity care system.

Pregnant woman: Well, I've got a [dating] scan on the 16th of September, and I don't see the midwife until after I've been to the scan. I would only see anybody if I did go for the triple test. That is the only time you see anybody in between ...

Interviewer: And how are you feeling about the scan?

Pregnant woman: I can't wait. I will feel better when I go to the scan, because I just can't wait to see something on that screen because you still don't feel like you are [pregnant]! (2.12, aged 26, declined screening, standard site, interview before screening would have been undertaken)

NICE guidelines (2003) recommend that women should have a booking appointment in the first trimester and a dating scan at 11-13 weeks. At the 
(standard) hospital referred to in the above quotation, women had contact with health professionals only for screening during a substantial phase of their pregnancy. Contact can confer incidental benefits including affirmation of pregnant status. Despite missing these benefits, the above respondent had rejected screening, not being willing to accept an increased risk of miscarriage from amniocentesis if she screened at higher risk.

Acceptance of higher risk candidacy entailed selective attention to chromosomal anomalies such as Down's syndrome.

Interviewer: Have you got any ideas of the sorts of things that the scan can show ...

Pregnant woman: Yeah, just any other sort of obvious defects, um, or, you know, from, I suppose, something like Spina Bifida ... But you always sort of think about Down's syndrome more, I think, because there's more press almost, kind of. People know more about it. (2.4, aged 30, private screening, standard site, interview after screening tests but before results known)

This woman had articulated the link between medical provision and risk selection. However, by defining the influence of medicine in terms of 'knowledge', she legitimated prioritising Down's syndrome over those of other conditions.

Acceptance of higher risk candidacy could be grounded in diverse projections about the future. For example, women who have ruled out diagnostic testing and termination may gamble that screening will generate a reassuring lower risk result (Heyman and Henriksen, 1998:183). Women who accepted screening on the basis that they would proceed to diagnostic testing if they screened at higher risk were required to gauge their feelings about a hypothetical contingency, an issue explored in the next quotation.

We were asked if we had talked about what to do if the result had come back high risk, whether or not we'd want an amnio or not. And we were explained that a lot of people at that stage say, 'Yes, definitely we want one', but then, once they look into it a little bit more, realise ... the risk of the amnio, they actually then choose not to have one. (2.15, aged 29, amniocentesis after higher risk screening, standard site, interview postscreening/diagnostic testing)

Despite being explicitly granted the licence to change their minds, this couple felt compelled to commit themselves to following through if necessary.

And she would have probably have sent us away ... [if] we'd have actually said, 'No. If the amnio comes back positive, we don't know what we'd do'. I'm not sure. She might have sent us away. She might have said, 'Look, I want you to decide on this before you proceed'. But we'd already decided, 
so it was pretty easy for her. (2.15, aged 29, amniocentesis after higher risk screening, standard site, interview post-screening/diagnostic testing)

Even when given advice to the contrary, women may feel that agreeing to screening entails acceptance of diagnostic testing if indicated.

Exceptionally, a woman could become an involuntary candidate for higher risk status when health professionals inadvertently screened her and informed her of the results even though she had declined screening, as illustrated below.

I was happy to have the blood tests done, and anything like that. But I wasn't prepared to have the Down's syndrome tests done anyway, and I had already made up my mind about that. (1.2, aged 21, involuntary higher risk screening, declined diagnostic testing, innovative site, post-screening interview)

This woman had decided to only accept serum screening for conditions such as HIV which could be treated. The error in this case may have resulted from the same test media, blood and ultrasound, being used for different purposes. Unfortunately, she had screened at higher risk and was trapped in this status until her baby was born. She could not 'unknow' the information which put her into the higher risk category (Williams, Alderson and Farsides, 2002).

\section{Rejection of Higher Risk Candidacy}

Women declined screening for various reasons, including rejection of its ultimate endpoint, abortion, challenging the risk selection implicit in the provision of screening for certain conditions, and avoidance of the anxiety arising from higher risk status. Ethical objections to abortion are illustrated below.

As I said earlier, we're Christians, and we've been praying to have a baby for years. So we're ready to accept what God gives us regardless, you know. And we know that he doesn't give bad gifts ... We are going to get a healthy baby. And whether the baby comes out right, we're going to have it. So why test it? (1.13, aged 35, declined screening, innovative site, interview after screening would have been undertaken)

Women who rejected pregnancy termination might still accept screening on the grounds of uncertainty reduction or in order to prepare themselves to care for a child with disabilities. However, this respondent, having ruled out abortion, saw no point in accepting screening.

Those who accepted screening took on an implicitly selective attitude towards risk concerns, as discussed above. Conversely, women who rejected screening could challenge its validity by declining to accept this attitude. 
And there's someone I work with, she had it [Down's syndrome screening] done. All her tests came back fine, and then her baby was born, and he's got, like, albinoism, you know. He's got problems with his sight. So, obviously, you can't detect everything. Obviously, the test isn't for all that. But it just goes to show, you never know what's going to be wrong. (2.12, aged 26, declined screening, standard site, interview before screening would have been undertaken)

Putting the available screening for chromosomal anomalies in a wider context reduced its perceived power to prevent the birth of a baby with health problems, thereby bolstering its rejection.

For some women, the risk of facing the worry associated with higher risk status influenced their decision to decline screening.

Pregnant woman: Then you could come back a high risk, and there could be nothing. There could be no risk at all ... And then if you have that [amniocentesis] done, there's a chance you can miscarry, so I would hate that to happen. That's why I think there's no point in having that first bit done because I worry about things as it is. (1.13, aged 35, declined screening, innovative site, interview after screening would have been undertaken)

As noted in relation to acceptance of higher risk candidacy, the rationale for her decision required this woman to make assumptions about how she would feel if she screened at higher risk.

\section{Living with Higher Risk Candidacy}

Screened women must live with uncertainty about their risk status until informed of their results. Contexts of risk interpretation associated with feeling anxious or not anxious are considered below.

Even the short wait required at the innovative site could generate considerable stress, as illustrated by the following consultation extract.

Midwife: The combined risk is what we look at, and the cut-off point on the combined risk is 1 in 300, and your combined is 1 in 9,922 ... 300 or less is a risk.

Pregnant woman: That's what l've got worried about. So I'm -

Midwife: Yes. The test in the screening is no way $100 \%$ guaranteed.

Pregnant woman: No, no. That looks fine to me though. That's a relief. I was getting a bit worried.

Midwife: Getting stressed?

Pregnant woman: Yes. It's my first, so I'm worrying about everything.

Midwife: Are you coming back to do classes with us? (Post-screening consultation 1.32, age not known, innovative site, transcript extract) 
This respondent took responsibility for the anxiety engendered by her higher risk candidacy, which she attributed to her neophyte status. The midwife's reference to 'stress' and her invitation to the woman to return to take classes reinforced service user ownership of anxiety about her risk status. The phrase ' 300 or less is a risk' nicely illustrates how an administratively determined cut-off for offering diagnostic testing could be transformed into an apparently natural property.

Women attending the standard site, who had to wait a week for screening results, did not necessarily experience high anxiety. The woman quoted below had been protected by optimistic assumptions which, unfortunately, were disconfirmed.

And that week wasn't spent worrying about it [screening] at all, to be honest. I was quite complacent in the fact that I thought everything would be okay. Then it came to Saturday and Sunday, and I sort of thought about it a little bit more, and I realised that Monday, Monday's the time that I might get a call ... She told me l'd come back as a high risk. And I was really upset, and I was crying, and she told me that I was 1 in 133. (2.15, aged 29, amniocentesis after higher risk screening, standard site, interview postscreening/diagnostic testing)

The drama of waiting for and receiving bad news perhaps contributed to this woman's distress. A sense of fatalism had partially protected the next respondent quoted from anxiety during the waiting period.

We did sort of speak about it quite a lot, but then I just tried to forget about it. Things like that you have no control over, I believe. So, if it's meant to be it's meant to be, do you know what I mean? So I don't feel it's worth sitting there pondering and worrying about it too much. I was a bit sort of anxious waiting for the results to come back, but then I'm like that with results of any sort. But they were fine when they came back. (2.11, aged 32, lower risk screening, standard site, interview post-screening)

These two examples illustrate ways in which women could manage the relatively long waiting period required at the standard site. Although the qualitative data did not reveal any examples of women experiencing extreme anxiety during the waiting period for screening results (as against diagnostic test results), a shorter waiting time reduced the need to adopt time management tactics.

\section{Living with Higher Risk Status}

Ascribing higher risk entails conversion of continuous probability values into a binary classification on the basis of an arbitrary cut-off. Differences in women's 
interpretations of and responses to this conversion are explored below. Women did not necessarily accept their higher risk classification, but some became highly distressed even if they appreciated its precautionary basis. Distress could be associated with self-blame, concealment of pregnancy and avoidance of identification with the fetus. Relationships with health professionals could become tense during this difficult waiting period.

The woman quoted below based rejection of the higher risk status ascribed to her on questioning the way in which probabilities were represented.

One in 61 was what my risk level was, or whatever. And my dad came back to me and said, 'That's only $2 \%$. It's a very, very small number'. If someone had said to me 2\%, I think I would have understood that more than 1 in 61. (2.14, aged 34, screened at higher risk but declined diagnostic test, standard site, interview post-screening)

This interpretation questions the cut-off used to differentiate higher and lower risk statuses, and contrasts with the acceptance of higher risk status discussed below. The next respondent quoted appeared to accept her risk $(1: 174)$ as both normal and high.

Um, well I think it was a normal result ... I don't know if l've got my figures right, but I thought 1 in 250, if there's 250 women there, one would have an abnormality. So 1 in 174, or whatever it was, I just imagined 174 women, and I could be that one person. So, yeah, it did seem quite a high risk, yeah. (2.16, aged 33, amniocentesis after higher risk screening, standard site, interview post-screening/diagnostic testing)

Communication even of a relatively 'normal' probability of an adverse event made it seem possible, and therefore 'quite high'. One woman had correctly reasoned that the cut-off of 1:250 was based on the precautionary principle, i.e. maximising detection.

And if it's more than 1 in 250, you're called a high risk, which I think is a terrible phrase for it, because high risk really means more than a 50\% chance, and it's not so. I actually came back with a 1 in 133, which is a one in point something percent chance, 0.75 I think we worked out the percent chance was, so hardly high risk. But, nevertheless, I don't suppose they take many chances. (2.15, aged 29, amniocentesis after higher risk screening, standard site, interview post-screening/diagnostic testing)

Despite identifying her risk status, which she labelled 'high' rather than 'higher', as precautionary, this woman experienced considerable distress during her three week waiting period (at the standard site) for her amniocentesis result. 
You would have to, you know, to stop something like that you just wanted so desperately um. So, yeah, very difficult to deal with. And I tried to stop myself thinking about that until we had the results, and thought what's the point in upsetting myself and maybe upsetting the baby ... And to a certain extent that worked as well ... Just kept busy as much as I could, um, because you'd just sit at home and dwell on it. And it takes forever. (2.15, aged 29, amniocentesis after higher risk screening, standard site, postnatal interview)

During the waiting period for diagnosis, the status of the desperately wanted pregnancy became indeterminate. Although women may be told that most diagnostic tests generate negative results, higher risk status can take on a reality of its own. This woman's concern was heightened by fear that her anxiety might be transmitted psychosomatically to the baby. She used time management techniques in order to get through a three week waiting period during which subjective time had slowed down.

Some women looked to their own conduct for an explanation of their higher risk status, compounding anxiety with self-blame.

I had like, I think it was 'flu ... I was taking painkillers for 10 days. I asked the midwife, 'Is it ... something to do with them?'. You just feel, you look for answers, don't you? ... And she said, 'No. It's just something that happens with genetics and chromosomes, nothing that you've done'. (1.1, aged 36, CVS after higher risk screening, innovative site, interview postscreening/diagnostic testing)

Her midwife had been able to reassure this respondent. As well as coping personally, women classified as at higher risk had to manage their relationships with others. The woman quoted below dealt with this issue through concealment, the requirements of which increased her distress.

I'm having problems at work, as to what to wear and things, and hope that nobody notices ... And emotionally, from lying to people basically ... I think I prefer to do it [conceal the pregnancy] particularly because we have decided that, if the results were very bad, we would terminate. I don't want to have to tell people that I've had a termination. (2.1, aged 40, amniocentesis after higher risk screening, standard site, interview post-screening/diagnostic testing)

This respondent's estimated probability of having a baby with chromosomal anomalies had been given as 1:119 based on her age which reduced to 1:249 after serum screening. The hospital used a probability of 1:250 as the cut-off for recommending diagnostic tests, which, she commented, was 'just one below not high risk'. Ironically, she had been catapulted into higher risk status by a screening result which reduced her prior age-based probability of carrying a 
fetus with chromosomal anomalies. This woman's acceptance of a borderline higher risk attribution was influenced by her belief that the test is 'only 60/40 accurate'. However, the '60/40 problem', as this respondent described it, refers to the $(40 \%)$ probability of woman screening at lower risk despite the presence of a fetal chromosomal anomaly. As she had screened at higher risk, this issue, of test sensitivity, did not apply to her case but, nevertheless, affected her understanding of being at higher risk.

Women placed in the higher risk category had to manage their relationships with health professionals whilst occupying this status. Two contrasting attitudes are illustrated below. The first respondent quoted valued the impartial but sympathetic thoroughness shown by the professional advising her.

No I was really pleased with the way that they handled everything. I thought the lady who spoke to us before the tests, or the amnio rather, we didn't feel hurried by her. She was open for any questions. She explained things in a very matter of fact way without seeming opinionated at all. She would give all the options without implying in her voice or how she said at which one she thought was the better one. (2.15, aged 29, amniocentesis after higher risk screening, standard site, postnatal interview)

Another respondent felt that the same hospital had failed to give her emotional support.

I know, it sounds silly really, but ... maybe they're understaffed at [standard site], but they don't really seem to give you the time that, to understand what it's like, what you're going through. (2.16, aged 33, amniocentesis after higher risk screening result, standard site, interview postscreening/diagnostic testing)

The next quotation depicts an unsympathetic response to a display of anxiety by a woman who was being scanned after being classified as at higher risk of trisomy 13 or 18.

And then I put my arm up over here, so then I shielded her [image of fetus] because I didn't want to see anything. And she said, 'Just keep your eye on the screen'. (1.1, aged 36, CVS after higher risk screening, innovative site, interview post-screening/diagnostic testing)

Service users will inevitably be treated differently, and will respond in diverse ways to the care they receive, particularly at a time of stress.

\section{Exit from Higher Risk Status}

Women lost their higher risk status when the chromosomal status of the fetus became known. The speed at which this transition was absorbed varied 
considerably. The respondent quoted below had moved easily from higher to no risk status.

I knew that it was definitely a normal result for Down's, and any other thing that they came back with would just be bad luck. There was no high risk of that. There was just as much chance of the other things as any other woman having a baby. So I relaxed. I was really pleased. (2.15, aged 29, amniocentesis after higher risk screening, standard site, postnatal interview)

One factor which might have contributed to the smoothness of this transition was her relief at a shortening of the waiting period for diagnosis. Initially informed that she would have to wait three weeks for diagnostics test results, this respondent then found that she could obtain partial results, including Down's syndrome for which she was at higher risk, within a week by purchasing private testing.

The transition from higher risk status sometimes required more than a diagnostic all clear, perhaps because the associated emotions could not be simply switched off, as the following quotation illustrates.

Although I do remember that the letter [confirming negative amniocentesis result] took a while to get here, and then I started to question myself a bit more ... It was nice to get the letter, quite strangely, I don't know why, to have it in writing, that guarantee again. (2.1, aged 40, amniocentesis after higher risk screening, standard site, postnatal interview)

The health professionals who gave the above respondent this good news reminded her of its limitations.

They said [at the time of giving amniocentesis result], 'You realise that this is not, it doesn't mean that you're going to have a healthy baby' ... They were obviously trying to say, 'This test is only to look at certain things, and don't think there's nothing else that could go wrong', kind of thing. Not very well put either. But, I think it was almost as if, if, then, we'd gone on to have a child with webbed feet, that we'd come back and say, 'Oh excuse me, I was guaranteed a perfect baby because I had an amnio'. (2.1, aged 40, amniocentesis after higher risk screening, standard site, postnatal interview)

This caution, which the above respondent found patronising, can be understood as a reminder that total exit from higher risk status with respect to one health problem does not imply the absence of other risks. As had respondent 2.15, quoted above, this woman appeared to re-engage with other risks following her exit from higher risk status for chromosomal anomalies.

Another respondent had not established attachment to the unborn child until a month after receiving the all clear. 
And one of the things that I ended up going for is actually saying that we'd like to know the sex of the baby if everything was ok ... And it probably took me about another month after having the results to actually finally think to myself, 'I am pregnant. I want to have this child'. (2.3, aged 40, amniocentesis on account of previous history, standard site, postnatal interview)

Knowing the sex of the baby would have established its personhood. The emotional barrier to engagement with the pregnancy, erected in case the adverse outcome associated with higher risk status came to pass, took time to break down.

Even grasping that a transition from higher risk to no risk status had taken place could prove problematic.

We kept thinking, 'Well, if it [diagnostic test] is clear ... there must, it can't be just that easy. There must be a next stage they're not telling us about'. It can't be, 'Oh yes, the results are clear. You've got a healthy baby'. There must be some other news they were going to break to us. (1.1, aged 36, amniocentesis after higher risk screening, innovative site, interview postscreening/diagnostic testing)

The difficulty experienced can be understood in terms of the tendency in riskoriented cultures to project uncertainty onto the external world. This projection may generate a belief that an individual placed into the higher risk category has a health problem. The above respondent found it difficult to understand that only the information about her baby, rather than the baby itself, had changed.

\section{DISCUSSION}

This paper has explored the process of chromosomal risk management at two maternity units operating 'standard' and 'innovative' screening systems. No claims are made about the typicality of the qualitative findings, which are designed more to illustrate 'what things "exist" than to determine how many such things there are' (Walker, 1985, p. 4).

As the UK and other countries move towards offering prenatal chromosomal screening universally, an increasing number of women will have to manage the psychosocial sequelae of higher risk status. Moreover, the advent of universal prenatal chromosomal screening reflects a wider shift towards a mode of health care in which concern for the individual case is replaced by a focus on the sorting of populations by risk indicators (Castel, 1991). Screening subjects its users to the risk of acquiring higher risk status. Its medical and psychosocial downsides have to be balanced against both the societal and personal gains arising from earlier detection of health problems and reassurance, for the majority, arising from the acquisition of lower risk status. 
The data presented above document the complex processes through which the provision of screening technology influences risk perceptions. Midwives and doctors (Heyman and Henriksen, 2001) can struggle to explain the complexities of probabilistic induction. Some women accept healthcare on the basis of trust, a process likely to be accentuated as an innovative form of healthcare becomes routinised (Press and Browner, 1997). Although the qualitative findings, involving small numbers of women on different screening pathways, did not generate evidence of differences between the standard and innovative sites, the survey findings cited above show that women who were processed through the latter more technically advanced and automated system were more likely to view prenatal chromosomal screening as routine and to accept screening. Acceptors may not appreciate that by accepting screening they become candidates for higher risk status. Qualitative data document the interpersonal processes underlying routinisation.

Responsibility for the 'quality control' of the fetus falls mainly on women (Rapp, 1999: 87). Decision-making about screening requires women to predict how they would feel in hypothetical future situations (Williams et al., in press). Santalahti et al. (1998) found that only half of the women who said that they would decline a termination after a positive amniocentesis result actually did so. This analysis raises troubling issues concerning the possibility of informed consent as advancing technology widens the scope for screening provision. Although its critics may struggle to suggest preferable alternatives, the limitations of the principle of informed consent, particularly its discounting of the social processes determining the menu of choices available and influencing individual decisions (Corrigan, 2003), need to be acknowledged. Health professionals need to clearly understand probabilistic reasoning and to appreciate the difficulty of communicating risk information effectively. As screening technology becomes more powerful, increasing amounts of information can be obtained from the same source, e.g. blood tests. Care needs to be taken to avoid patients being inadvertently being given information about their risk status, for example in patient-held records, which they have opted not to receive.

Screening creates a new, usually transient, role of living with higher risk status. The present study illustrates two challenges to the validity of this attribution. Firstly, the limited scope of chromosomal screening in relation to the overall range of pregnancy-related health problems could be noted. This challenge undermines the tacit selection of risks as targets for concern which is embedded in the provision of particular risk management systems. Secondly, the transformation of quantitative probabilities into the qualitative categories of 'higher' versus 'lower' risk could be questioned.

Those study participants who accepted higher risk status experienced varying degrees of distress during the waiting period for diagnostic test results, a time of arguably tentative pregnancy (Rothman, 1994). They treated their screening 
result as 'positive', i.e. indicative of a likely problem, even when they clearly understood that this categorisation arose from being above a precautionary threshold. Such reactions should not be dismissed as mere misunderstanding, a view expressed by $80 \%$ of a sample of obstetricians (Green, 1994). More attention needs to be given to the needs of women who screen at higher risk (Green et al., 2004), and primary care professionals need to be more engaged in supporting them (Statham, Solomou and Green, 2003). Women will occasionally acquire higher risk status unintentionally through receiving results from screening tests which they had declined. Such accidents may become more likely as the same medium, e.g. blood tests or scans, are used for more and more screening purposes as technology advances. Women who acquire higher risk status inadvertently may be in particular need of support, and their inadvertent acquisition of higher risk status should be noted on their records. Exiting from higher risk status was not always straightforward. Similarly, Weinans et al. (2000) found that $13 \%$ of the women they surveyed continued to feel anxious after amniocentesis had ruled out chromosomal anomalies.

The operation of screening systems marks some service users as at higher risk, and therefore in need of further investigation. These service users, who cannot be identified in advance, pay the price of living with higher risk status so that all can be given the option of taking preventative action if the conditions being screened for are eventually identified. 


\section{REFERENCES}

Aitken, D.A., Crossley, J.A., \& Spencer, K. (2002). Screening for neural tube defects and aneuploidy. In D.L. Romoin, J.M. Connor, R.E. Pyeritz \& B.R. Korf (Eds.), Principles and Practice of Medical Genetics. London: Churchill Livingstone.

Bindra, R., Heath, V., Liao, A., Spencer, K., \& Nicolaides, K.H. (2002). One-stop clinic for assessment of risk for trisomy 21 at $11-14$ weeks: a prospective study of 15030 pregnancies. Ultrasound in Obstetrics \& Gynecology, 3, 219-225.

Canini, S., Prefumo, F., Famularo, L., Venturini, .L., Palazzese, V., \& De, B.P. (2002). Comparison of first trimester, second trimester and integrated Down's syndrome screening results in unaffected pregnancies. Clinical Chemistry \& Laboratory Medicine, 40, 600-603.

Castel, R. (1991). From dangerousness to risk. In G. Burchell, C. Gordon and P. Miller (Eds.), The Foucault Effect. London: Harvester Wheatsheaf.

Corrigan, O. (2003). Empty ethics: the problem with informed consent. Sociology of Health \& Illness, 25, 768-792.

Fredouille, C., Piercecchi-Marti, M.D., Liprandi, A., Duyme, M., Gonzales, M., Bigi, N. (et al) (2002). Linear insertion of atrioventricular valves without septal defect: a new anatomical landmark for Down's syndrome? Fetal Diagnosis \& Therapy, 17,188-92.

Gigerenzer, G. (2003). Reckoning with Risk: Learning to Live with Uncertainty. Harmondsworth: Penguin.

Gigerenzer, G. and Selten, R. (2001) Rethinking rationality. In G. Gigerenzer and R. Selten (Eds.) Bounded Rationality: The Adaptive Toolbox. (pp. 1-12). Cambridge MA: MIT Press.

Goel V., Glazier R., Summers A. and Holzapfel S. (1998) Psychological outcomes following maternal serum screening: A cohort study, Canadian Medical Association Journal, 159, 651-656.

Green, J.M. (1994). Serum screening for Down's syndrome: Experiences of obstetricians in England and Wales. British Medical Journal, 309, 769-72.

Green, J.M., Hewison, J., Bekker, H.L., Bryant, L.D. and Cuckle, H.S. (2004). Psychosocial aspects of gender screening of pregnant women and newborns: A systematic review. Health Technology Assessment, 8, Whole number 33. 
Greenland, S., \& Robbins, J. (1994). Invited commentary: Ecological studies: biases, misconceptions and counterexamples. American Journal of Epidemiology, 139, 747-760.

Heyman, B. (2005). Health Risk Escalators. In R. Bibace, J. Laird and J. Valsiner (Eds.) Science and Medicine in Dialogue: Thinking Through Particulars and Universals. Westport, Ct.: Greenwood Publishing Group.

Heyman, B. \& Henriksen, M. (2001). Risk, Age and Pregnancy: A Case Study of Prenatal Genetic Screening and Testing. Basingstoke: Palgrave.

Henriksen, M. \& Heyman, B. (1998). Being old and pregnant. In B. Heyman (Ed.) Risk, Health and Health Care: A Qualitative Approach. (pp 171-186). London: Edward Arnold.

Heyman, B., Henriksen, M. \& Maughan, K. (1998). Probabilities and Health Risks: A Qualitative Approach. Social Science \& Medicine, 9, 1295-1306.

Lewando Hundt, G., Sandall, J., Spencer, K., Williams, C. \& Heyman B. (2005). Social Implications of One Stop First Trimester Antenatal Screening. London: Unpublished Report, ESRC Project.

Leithner, K., Maar, A., Fischer-Kern, M., Hilger, E., Loffler-Stastka H., \& Ponocny-Seliger, E. (2004). Affective state of women following a prenatal diagnosis: predictors of a negative psychological outcome. Ultrasound in Obstetrics \& Gynecology, 23, 240-246.

NICE (2003). Antenatal Care for the Pregnant Woman. http://www.nice.org.uk .

Press, N. \& Browner, C.H. (1997). Why women say yes to prenatal diagnosis. Social Science \& Medicine, 45, 979-989.

Rapp, R. (1999). Testing Women, Testing the Foetus. New York: Routledge.

Ritchie, J., \& Lewis, J. (2003). Qualitative Research Practice: A Guide for Social Science Students and Researchers. London: Sage.

Robinson, W.S. (1950). Ecological correlations and the behaviour of individuals. American Sociological Review, 15, 351-357.

Rothman, K.B. (1994). The tentative pregnancy: Then and now. Rapp R. (1994). Women's responses to diagnosis. In K.H. Rothenberg and E.J. Thomson (Eds.), Women and Prenatal Testing: Facing the Challenges of Genetic Technology. Columbus: Ohio State University Press. 
Santalahti, P., Hemminki, E., Aro, A.R. \& Helenius, H. (1998). Participation in prenatal screening tests and intentions concerning selective termination in Finnish maternity care. Foetal Diagnosis and Therapy, 14, 71-79.

Seth, J. \& Ellis, A.R. (1994). The United Kingdom National External Quality Assessment Scheme for screening for Down's syndrome. In J.G. Grudzinskas, T. Chard, M. Chapman and H. Cuckle (Eds.), Screening for Down's Syndrome. Cambridge: Cambridge University Press.

Spencer, K. (2001). Age related detection and false positive rates when screening for Down's syndrome in the first trimester using fetal nuchal translucency and maternal serum free beta-hCG and PAPP-A. The British Journal of Obstetrics \& Gynaecology, 108, 1043-6.

Statham, H., Solomou, W. \& Green, J.M. (2003). Communication of prenatal screening and diagnosis results to primary-care health professionals. Public Health, 117, 348-57.

Tabor, A., Madsen, M., Obel, E.B., Philip, J., Bang, J., Norgaard-Pedersen, B. (1986). Randomised controlled trial of genetic amniocentesis in 4606 low-risk women. Lancet 1 (8493), 1287-93.

Wald, N.J., Watt, H.C. \& Hackshaw, A.E. (1999). Integrated screening for Down's syndrome on the basis of tests performed during the first and second trimesters. New England Journal of Medicine, 341, 461-467.

Walker, R. (1985). An introduction to applied qualitative research. In R. Walker (Ed.) Applied Qualitative Research. Aldershot: Gower.

Weinans, M.J., Kooij, L., Muller, M.A., Bilardo, K.M., Van Lith, J.M., \& Tymstra, T. (2004). A comparison of the impact of screen-positive results obtained from ultrasound and biochemical screening for Down syndrome in the first trimester: a pilot study. Prenatal Diagnosis, 24, 347-51.

Williams, C., Alderson, P. \& Farsides, B. (2002). 'Drawing the line' in prenatal screening and testing: Health practitioners' discussions. Health, Risk \& Society, 4, 51-75.

Williams, C., Sandall, J., Lewando Hundt, G., Grellier, R., Heyman, B. \& Spencer, K. (In press) Women as moral pioneers? Experiences of first trimester antenatal screening. Social Science \& Medicine. 